\title{
Avaliação da diversidade alfa de Passalidae (Coleoptera: Scarabaeoidea) na Amazônia Colombiana (Parque Nacional Natural La Paya, Putumayo)
}

\author{
Larry JIMÉNEZ-FERBANS ${ }^{1}$, Germán AMAT-GARCÍA²
}

RESUMO

Este trabalho apresenta um inventário da fauna de Passalidae da regiāo sudeste do Parque Nacional Natural La Paya, sendo a primeira coleta deste grupo de insetos para o Estado colombiano de Putumayo. As coletas, de indivíduos adultos, foram feitas durante os dias 9 a 16 de fevereiro de 2008, em percursos livres, nos quais foram revisados 80 troncos em decomposiçáo. A porcentagem de troncos colonizados por passalídeos foi de $40 \%$, nos quais foram coletados 225 indivíduos pertencentes a duas tribos, quatro gêneros e 16 espécies, representando entre $73-96 \%$ da riqueza estimada por curvas de acumulação de espécies. A tribo com maior riqueza foi Passalini, enquanto o gênero com maior diversidade foi Passalus Fabricius, 1792 com 12 espécies. A diversidade local do grupo estudado é alta, em comparação a outros registros obtidos na região neotropical.

Palavras-Chave: Passalídeo, diversidade, Putumayo

\section{Alpha diversity assessment of Passalidae (Coleoptera: Scarabaeoidea) in the Colombian Amazon (La Paya National Natural Park, Putumayo)}

\begin{abstract}
It is presented an inventory of the Passalidae fauna from Southeastern National Natural Park La Paya, Colombia. This corresponds to the first survey of this family for the Putumayo region. The collections were conducted from February 9-16th 2008, along forest tracks, assessing a total of 80 decaying logs. In general, $40 \%$ of the logs were colonized by passalids. The 225 individuals collected belong to two tribes, four genera and 16 species, which represent between 73 to $96 \%$ of the richness estimated by species accumulation curves. The tribe with the greatest richness was Passalini, while the most diverse genus was Passalus Fabricius, 1792 with 12 species. The local diversity of the study area is among the highest when compared to other Neotropical areas.
\end{abstract}

KEYWORDS: Passalid beetles, diversity, Putumayo

1 Instituto de Ecología. larryjimenezferbans@gmail.com

2 Universidad Nacional de Colombia. gdamatg@unal.edu.co 


\section{INTRODUÇÃO}

A Amazônia colombiana, com $300.092 \mathrm{~km}^{2}$ de extensão, representa $30 \%$ da área do país, ocupando territórios dos Estados de Guainía, Guaviare, Vaupés, Putumayo, Caquetá e Amazonas. Nesta importante região do país, têm sido realizados diversos estudos sobre a fauna de Hymenoptera e Coleoptera (Escobar 2000; Fernández 2001; Pulido et al. 2003; Amat-García e Reyes-Castillo 2007; Guerrero 2009; Sarmiento-Garcés e Amat-García 2009). No entanto, devido a sua grande extensão territorial, ainda existem áreas inexploradas para o estudo da fauna de coleópteros. A maioria dos estudos conhecidos foi direcionada aos coleópteros coprófagos (Scarabaeidae, Scarabaeinae). O conhecimento faunístico de outros grupos (p. ex.: Chrysomelidae, Curculionidae, Lucanidae, Staphylinidae, Trogidae) é escasso ou nulo.

Os Passalidae são insetos subsociais em que os imaturos e adultos desenvolvem-se no interior de troncos em decomposição, onde se alimentam. A família tem elevada concentração de espécies nas zonas úmidas tropicais, com endemismos em zonas de montanha, sendo um grupo de besouros recentemente inserido em propostas de delimitação de áreas prioritárias para a conservação (Schuster et al. 2000). A família está formada por 680 espécies conhecidas, com distribuição Pantropical. As espécies neotropicais pertencem à Passalinae, tribos Proculini e Passalini (Boucher 2005). Para a Colômbia são conhecidas 88 espécies alocadas em 10 gêneros (Amat-García et al. 2004). De acordo com trabalhos recentes, o número de espécies pode chegar a 100 (Boucher 2005; Amat-García e Reyes-Castillo 2007; Jiménez-Ferbans e Amat-García 2009).

O plano de inventário das espécies de Passalidae, na Colômbia, tem avançado significativamente nos últimos anos; e na Amazônia Colombiana já foram realizados vários trabalhos, como os de Amat-García e Reyes-Castillo (1996); Boucher (2005); Reyes-Castillo et al. (2005); Amat-García e Reyes-Castillo (2007). Entretanto, não há resultados sistemáticos de campo que respondam a protocolos de amostragem local (diversidade alfa) intensivos, na região amazônica, razão pela qual se apresentam os resultados sobre a diversidade alfa de besouros passalídeos.

\section{MATERIAL E MÉTODOS}

De acordo com o esquema biogeográfico apresentado por Morrone (2001), a Amazônia colombiana está formada por duas províncias: Imerí (área interfluvial do Alto Amazonas), localizada nas regióes sul da Venezuela, sul da Colômbia, nordeste do Peru e norte do Brasil. A outra província é Napo, formada pela área interfluvial de Putumayo-Caquetá e, que corresponde ao nordeste do Peru, sudeste da Colômbia e leste do Equador. As coletas foram realizadas na zona sudeste do Parque Nacional Natural La Paya, inserido na segunda província mencionada. $\mathrm{O}$ parque possui uma extensão de 422.000 hectares e as coletas foram realizadas em uma área de 120 hectares de floresta primária, formada por florestas de terra firme e várzea, sobre um gradiente de altitude homogêneo entre 200 e $300 \mathrm{~m}$. A área de estudo está localizada a 14 $\mathrm{km}$ à nordeste do lugarejo Bajo Causante $\left(00^{\circ} 04^{\prime} 37,9^{\prime \prime} \mathrm{S}\right.$ $\left.074^{\circ} 59^{\prime} 19,4^{\prime \prime} \mathrm{W}\right)$.

As coletas foram realizadas em fevereiro de 2008, através de percursos onde foram avaliados 80 troncos em estado de decomposição, cortados com machado. Somente adultos foram coletados e o material foi preservado em álcool etílico $70 \%$. Os exemplares foram identificados ao nível de gênero, com utilização da chave de Schuster e Cano (2005), e ao nível de espécie com a ajuda das descriçóes originais e através de comparação com o material depositado na Coleção de Entomologia do Instituto de Ciências Naturais da Universidade Nacional da Colômbia. O material coletado está depositado na Coleção Entomológica da Universidad del Magdalena (CEUM) - Santa Marta, Colômbia.

\section{ANÁLISE DE DADOS}

Com o objetivo de avaliar a representação do esforço de amostragem, foram geradas curvas de acumulação de espécies de forma que a ordem das amostras foi aleatorizada em 500 vezes, no programa EstimateS, versão 6.1 (Colwell 2005). Além disso, foram usados os estimadores Chao 1 e Jacknife 1, que permitem calcular o número esperado para uma determinada zona, baseados nas características da amostragem (Moreno 2001).

\section{RESULTADOS E DISCUSSÃO}

Foram coletados 225 indivíduos de 16 espécies (Tabela 1). Embora a curva de acumulação de espécies não mostre uma distribuição claramente assintótica, o nível da amostragem pode ser considerado como aceitável segundo os estimadores empregados, que indicam um nível de representação de 7396\% (Figura 1). Assim, o decréscimo de espécies com um exemplar (singletons) sugere uma boa coleta. O número de espécies registradas foi alto quando comparado com os resultados obtidos por Mouzinho e Fonseca (1998), os quais revisaram 501 troncos na Amazônia Central, encontrando nove espécies de passalídeos, enquanto Jiménez-Ferbans et al. (2010) encontraram uma riqueza para o Caribe Colombiano de 14 espécies em 630 troncos.

A espécie mais abundante foi Passalus rhodocantopoides (Kuwert 1891) com 91 indivíduos (40\%), contrariando as informaçôes de Amat-García e Reyes-Castillo (2007), que citaram esta espécie como amazônica e com certa raridade local. A maior abundância de $P$. rhodocantopoides corresponde 
Tabela 1 - Abundância de adultos por espécie de Passalidae coletados no setor sudeste do Parque Nacional Natural La Paya.

\begin{tabular}{|c|c|}
\hline Espécie & Abundância \\
\hline Paxillus cf. camerani (Rosmini, 1902) & 2 \\
\hline Spasalus crenatus (MacLeay, 1819) & 2 \\
\hline Passalus (Pertinax) convexus Dalman, 1817 & 2 \\
\hline Passalus (Pertinax) epiphanoides (Kuwert, 1891) & 31 \\
\hline $\begin{array}{l}\text { Passalus (Pertinax) aff pseudoconvexus Boucher, } \\
1990\end{array}$ & 1 \\
\hline $\begin{array}{l}\text { Passalus (Pertinax) rhodocanthopoides (Kuwert, } \\
\text { 1891) }\end{array}$ & 91 \\
\hline Passalus (Passalus) abortivus Percheron, 1835 & 13 \\
\hline $\begin{array}{l}\text { Passalus (Passalus) barrus Boucher \& Reyes- } \\
\text { Castillo, } 1991 .\end{array}$ & 1 \\
\hline Passalus (Passalus) coniferus Eschscholtz, 1829 & 3 \\
\hline $\begin{array}{l}\text { Passalus (Passalus) glaberrimus Eschscholtz, } \\
1829\end{array}$ & 15 \\
\hline $\begin{array}{l}\text { Passalus (Passalus) interstitialis Eschscholtz, } \\
1829\end{array}$ & 35 \\
\hline $\begin{array}{l}\text { Passalus (Passalus) punctiger Lepeletier \& } \\
\text { Serville, } 1825\end{array}$ & 2 \\
\hline Passalus (Passalus) sp1 & 7 \\
\hline Passalus (Passalus) sp2 & 1 \\
\hline Veturius sp1 & 7 \\
\hline Veturius sp2 & 12 \\
\hline Total & 225 \\
\hline
\end{tabular}

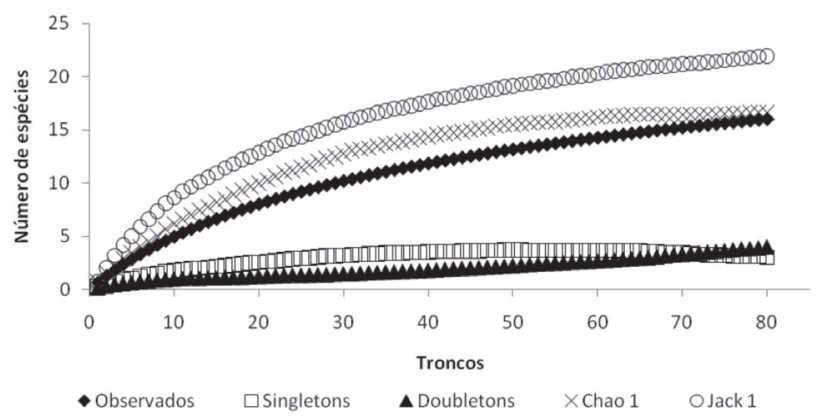

Figura 1 - Curvas de acumulação de espécies de passalídeos para a zona sudeste do P.N.N. La Paya

ao censo de quatro grupos familiares com 17-27 indivíduos, encontrados em igual número de troncos. Nesse sentido, o número de troncos colonizados por $P$. rhodocantopoides (6) foi inferior ao colonizado por Passalus (Pertinax) epiphanoides (Kuwert, 1891) (14), que apresentou a maior frequência de troncos colonizados. Em geral, os troncos colonizados por passalídeos representaram $40 \%$ do total amostrado, o que indica um bom nível de aproveitamento do recurso. Este valor é similar ao encontrado por Jiménez-Ferbans et al. (2010), que obtiveram entre $38 \%$ e $45 \%$ do aproveitamento do recurso para zonas úmidas do Caribe colombiano, e superior ao encontrado por Mouzinho e Fonseca (1998), 19\% para sistemas degradados na Amazônia brasileira. É muito provável que o estado de conservação e o alto nível de cobertura da folhagem facilitem as condiçóes de umidade ótimas para o estabelecimento dos grupos familiares de Passalidae.

A maioria dos troncos ocupados (53\%) tinha uma espécie. $\mathrm{O}$ número máximo de espécies encontradas em um mesmo tronco foi três, o que ocorreu em apenas cinco dos troncos estudados. Essa quantidade máxima de espécies por tronco é a mesma registrada por Mouzinho e Fonseca (1998), enquanto Castillo e Reyes-Castillo (1997) registraram quatro espécies. Além disso, $72 \%$ dos troncos ocupados tinham abundância de oito ou menos indivíduos, inferior à média de 15 indivíduos por tronco encontrado por Castillo e Lobo (2004) em ambientes tropicais do México.

A fauna de passalídeos do PNN La Paya é composta por duas tribos, quatro gêneros e 16 espécies. A tribo com maior riqueza foi Passalini e o gênero com maior diversidade foi Passalus Fabricius, 1792 com 12 espécies, resultado compreensível por ser este gênero, em especial o subgênero Passalus, particularmente, rico e abundante na região amazônica. Ao mesmo tempo, Proculini, tipicamente da América Central, está representada na região por Veturius Kaup, 1871.

Antes deste estudo sete espécies de passalídeos foram registradas para o Estado de Putumayo (Amat-García et al. 2004; Boucher 2005), a saber: Passalus (P.) coniferus Eschscholtz, 1829; Passalus (Pertinax) convexus Dalman, 1817; Paxillus leachi MacLeay, 1819; Veturius caquetaensis Boucher, 1988; Veturius sinuosus (Drapiez, 1820); Veturius standfussi Kwert, 1891 e Veturius yahua Boucher, 2005. Considerando que este trabalho representa a primeira tentativa sistemática de estudar a fauna de Passalidae de Putumayo, a diversidade alfa da zona estudada é alta quando comparada com a registrada para outros ambientes (Tabela 2). Nesse sentido, Amat-García e Reyes-Castillo (2007) encontraram uma diversidade de 14 espécies em um período de quatro anos de coletas no Estado do Amazonas (Colômbia), que corresponde ao valor da diversidade gama. Este valor de riqueza é equivalente as 23 espécies pelo estimador Chao 1, só para a área amostrada no PNN La Paya (diversidade alfa). Além disso, se as 16 espécies coletadas no presente estudo forem adicionadas as cinco citadas por outros autores (Reyes-Castillo e AmatGarcía 2003; Boucher 2005) para outras zonas do estado de Putumayo, teríamos uma riqueza equivalente à diversidade gamma já conhecida para o Estado do Amazonas.

A comunidade de passalídeos de Putumayo está formada principalmente por elementos de origem guiano- amazônicos, alguns dos quais chegam até as zonas baixas do norte da Colômbia (Spasalus crenatus (Mac Leay, 1819); Passalus 
Tabela 2 - Riqueza de espécies de Passalidae para várias zonas do Neotrópico. bh-T: Floresta úmida tropical, bs-T: Floresta seca tropical, bmh-MB: Floresta muito úmida montana baixa, bh-PM: Floresta úmida Premontana, BTP: Floresta tropical perenifólia.

\begin{tabular}{lcccc}
\hline Zona & Diversidade & Formação vegetal & Riqueza & Referência \\
\hline PNN La Paya & alfa & bh-T & 16 & Presente estudo \\
Sierra Nevada de Santa Marta-Colombia & gamma & bs-T - bmh-MB & 15 & Jiménez-Ferbans \& Amat-García (2009) \\
Serranía del Perijá-Colombia & gamma & bs-T - bh-PM & 6 & Jiménez-Ferbans \& Amat-García (2009) \\
Amazonas-Colombia & gamma & bh-T & 24 & Amat-García \& Reyes-Castillo (2007) \\
Amazonia central-Brasil & alfa & bh-T & 9 & Mouzinho \& Fonseca (1998) \\
Los Tuxtlas-México & alfa & BTP & 15 & Castillo \& Reyes-Castillo (1997) \\
Sabana de Bogotá-Colombia & alfa & bmh-MB & 8 & Amat-García \& Reyes-Castillo (2002) \\
Manaus & gamma & bh-T & 24 & Reyes-Castillo et al. (2005) \\
\hline Tambopata & gamma & bh-T & 17 & Reyes-Castillo et al. (2005) \\
\hline
\end{tabular}

bh-T: tropical rainforest, BST: tropical dry forest, BMH-MB: sub-montane wet forest,

bh-PM: pre-montane rainforest, BTP: tropical evergreen forest.

interstitialis Eschscholtz, 1829; Passalus coniferus Eschscholtz, 1829; Passalus punctiger Lepeletier e Serville, 1825). Exceto P. rhodocantopoides, que na Colômbia estava registrada para o Estado do Amazonas, as espécies do subgênero Pertinax Kaup, 1869 apresentam registro até a Cordilheira dos Andes. Assim, pela sua localização na província de Napo, onde convergem elementos amazônicos e andinos, no Estado de Putumayo encontram-se também elementos faunísticos tipicamente andinos como Veturius standfussi Kuwert, 1891 (Boucher 2005).

\section{AGRADECIMENTOS}

À Corporação Ecozóica pelo financiamento do estudo e à comunidade indígena Siona pelo apoio logístico, durante as coletas no campo. Ao Hector Gasca e Andrea Carneiro pela tradução do manuscrito para o Português e a Natalia Cortés e Germán Forero pela ajuda com o abstract.

\section{BIBLIOGRAFIA CITADA}

Amat-García, G.; Reyes-Castillo, P. 1996. The passalid beetles (Coleoptera: Passalidae) of Colombia II: geographic and altitudinal distribution, p. 75-92. In: Andrade-C., M.; Amat, G.; Fernández, F. (Eds). 2009. Insect of Colombia: selected studies. Academia Colombiana de Ciencias Exactas, Físicas y NaturalesCentro Editorial Javeriano. Bogotá D.C. (in Spanish, with abstract in English).

Amat-García, G.; Reyes-Castillo, P. 2007. The passalid beetles (Coleoptera: Scarabaeoidea: Passalidae) from Amazonas Departament, Colombia. Caldasia, 29: 329-354 (in Spanish, with abstract in English).

Amat-García, G.; Blanco-Vargas, E.; Reyes-Castillo, P. 2004. A Species List of Passalid Beetles (Coleoptera: Passalidae) of Colombia. Biota Colombiana, 5: 173-182 (in Spanish and English).
Boucher, S. 2005. Evolution and phylogeny of Passalidae (Scarabaeoidea). Annales de la Société Entomologique de France, 41: 1-603 (in French, with abstract in English).

Castillo, M.L.; Reyes-Castillo, P. 1997. Passalidae, p. 293-298. In: González, E.; Dirzo R.; Voght, R. (Eds.). Natural History from "Los Tuxtlas". UNAM, México (in Spanish).

Castillo, M.L.; Lobo, J.M. 2004. A comparison of Passalidae (Coleoptera, Lamellicornia) diversity and community structure between primary and secondary tropical forest in Los Tuxtlas, Veracruz, México. Biodiversity and Conservation, 13: 1257-1269.

Colwell, R.K. 2005. EstimateS: Statistical estimation of species richness and shared species from samples. Version 7.5. (http:// viceroy.eeb.uconn.edu/estimates). Acesso em 15/12/2009.

Escobar, F. 2000. Dung beetles diversity (Scarabaeidae: Scarabaeinae) in mosaic of habitats in the Natural Reserve Nukak, Guaviare, Colombia. Acta Zoologica Mexicana, 79: 103-121 (in Spanish, with abstract in English).

Fernández, F. 2001. Ants of Colombia IX. New species of Lenomyrmex (Formicidae: Myrmicinae). Revista Colombiana de Entomologia, 27: 201-204 (in Spanish, with abstract in English).

Guerrero, R. 2009. First record of the ant genus Myrcidris (Formicidae: Pseudomyrmecinae) from Colombia. Revista Colombiana de Entomología, 35: 103-104.

Jiménez-Ferbans, L.; Amat-García, G. 2009. The passalid beetles (Coleoptera: Scarabaeoidea: Passalidae) from Caribbean region of Colombia. Caldasia, 31: 155-173 (in Spanish, with abstract in English).

Jiménez-Ferbans, L.; Amat-García, G.; Reyes-Castillo, P. 2010. Diversity and distribution patterns of Passalidae (Coleoptera Scarabaeoidea) in the Caribbean Region of Colombia. Tropical Zoology, 23: 147-164.

Moreno, C.E. 2001. Methods for measuring biodiversity. M\&TManuales y Tesis Sociedad Entomológica Aragonesa, vol. I. Zaragoza. 84 pp. (in Spanish). 
Morrone, J.J. 2001. Biogeography of Latin America and Caribbean. Manuales \& Tesis, Sociedad Entomológica Aragonesa, vol. III. Zaragoza. 148 pp. (in Spanish).

Mouzinho, J.; Fonseca, C. 1998. Contribution to the study of passalid beetles (Coleoptera, Scarabaeoidea, Passalidae) in a land area of the Central Amazon. Acta Zoologica Mexicana, 73: 19-44 (in Portuguese, with abstract in English).

Pulido, L.; Riveros, R.; Gast, F.; von Hildebrand, P. 2003. Dung beetles (Coleoptera: Scarabaeidae: Scarabaeinae) from de Serranía Chiribiquete National Natural Park, Caquetá, Colombia (Part I), p. 51-58. In: Onore, G.; Reyes-Castillo, P.; Zunino, M. (Eds.). Scarabaeoidea of Latin America: state of knowledge. Monografías Tercer Milenio vol. III. Sociedad Entomológica Aragonesa, Zaragoza (in Spanish, with abstract in English).

Reyes-Castillo, P.; Amat-García, G. 2003. Passalidae (Coleoptera) of Colombia, p. 35-50. In: Onore, G.; Reyes-Castillo, P.; Zunino, M. (Eds). Project of Iberoamerican Biogeography and Systematic Entomology Network PrIBES 2003. Monografías tercer milenio vol. III. Sociedad Entomológica Aragonesa, Zaragoza (in Spanish, with abstract in English).
Reyes-Castillo, P.; Amat-García, G.; Fonseca, C.R.V. 2005. Parsimony analysis of endemicity of Passalidae (Coleoptera: Scarabaeoidea) in the Amazon subregion, p. 461-467. In: Llorente-Bousquets, J.; Morrone, J.J. (Eds.). Biogeographical regions in Latin America and related topics. Las Prensas de Ciencias, UNAM, México (in Spanish).

Sarmiento-Garcés, R.; Amat-García, G. 2009. Beetles Dichotomius Hope, 1838 (Scarabaeidae: Scarabaeinae) in the Colombian Amazon. Revista de la Academia Colombiana de Ciencias, 33: 291-302 (in Spanish, with abstract in English).

Schuster, J.; Cano, E.; Cardona, C. 2000. A simple method to prioritize the conservation of cloud forests of Guatemala, using Passalidae (Coleoptera) as indicator organisms. Acta Zoológica Mexicana, 80: 197-209 (in Spanish, with abstract in English).

Schuster, J.; Cano, E. 2005. Key to american genera of Passalidae. (www-museum.unl.edu/research/entomology/Guide/ Scarabaeoidea/Passalidae/ Passalidae-Key/PassalidaeK.html). Acesso em 27/07/2008.

Recibido Em 05/04/2010

Aceito Em 17/07/2010 